\title{
Gamificação: expectativa educativa, impacto na saúde
}

\author{
Elisabete Zimmer Ferreira \\ Enfermeira, Mestre em Enfermagem. Doutoranda do Programa de Pós-graduação em Enfermagem da \\ Universidade Federal do Rio Grande (FURG), Rio Grande, RS, Brasil \\ 凶 elisabetezimmer@yahoo.com.br \\ Adriane Maria Netto de Oliveira \\ Enfermeira, Doutora em Enfermagem. Professora Associada da Escola de Enfermagem da \\ Universidade Federal do Rio Grande (FURG), Rio Grande, RS, Brasil

\begin{abstract}
Aline Neutzling Brum
Bióloga, Pós-doutora em Enfermagem. Professora Visitante da Escola de Enfermagem da Universidade Federal do Rio Grande (FURG), Rio Grande, RS, Brasil
\end{abstract} \\ Mara Regina Santos da Silva \\ Enfermeira, Doutora em Enfermagem. Professora Associada da Escola de Enfermagem da \\ Universidade Federal do Rio Grande (FURG), Rio Grande, RS, Brasil

\section{Luciano Garcia Lourenção} \\ Enfermeiro, Doutor em Ciências da Saúde. Professor Titular-Livre da Escola de Enfermagem da \\ Universidade Federal do Rio Grande (FURG), Rio Grande, RS, Brasil
}

\section{Resumo:}

Os dispositivos tecnológicos fazem parte do cotidiano da sociedade e marcam presença também no ambiente escolar. Diante disso, a gamificação tem sido utilizada como alternativa às abordagens tradicionais da educação. Assim, realizou-se um ensaio teórico com o objetivo de refletir sobre a gamificação na educação e o risco do uso desmedido de jogos digitais para a saúde dos adolescentes. Nesta reflexão discorre-se sobre o uso de jogos digitais no contexto educacional, o risco por trás dos jogos digitais e estratégias para minimizar danos à saúde, decorrentes da utilização dos jogos. Concluiu-se que a gamificação demonstra ser um excelente catalizador da aprendizagem. No entanto, se for estimulada sem moderação, pode gerar um fascínio pelo jogo, desencadeando sentimentos de ansiedade, agressividade e de compulsão. Portanto, é necessário que os atores sociais envolvidos direta ou indiretamente na formação dos adolescentes, como a família, a comunidade escolar e os profissionais de saúde estejam atentos a possíveis mudanças no comportamento dos adolescentes e estruturem ações, estratégias e políticas de atenção à saúde, focadas no uso dos jogos digitais pelos adolescentes.

Palavras-chave: Educação, Jogos de vídeo, Materiais de ensino, Saúde do adolescente.

\section{Gamification: educational expectation, impact on health}

\begin{abstract}
:
Technological devices are part of daily life of society and are also present in school environment. Therefore, gamification has been used as an alternative to traditional approaches to education. Thus, a theoretical essay was conducted with objective of reflecting on gamification in education and the
\end{abstract}


risk of excessive use of digital games for health of adolescents. This reflection discusses the use of digital games in educational context, the risk behind digital games and strategies to minimize damage to health, arising from use of games. It was concluded that gamification proves to be an excellent catalyst for learning. However, if stimulated without moderation, it can generate a fascination for game, triggering feelings of anxiety, aggression and compulsion. Therefore, it is necessary that social actors directly or indirectly involved in education of adolescents, such as family, school community and health professionals, be aware of possible changes in behavior of adolescents and structure actions, strategies and policies of health care, focused on use of digital games by teenagers.

Keywords: Education, Video games, Teaching materials, Adolescent health.

\section{Gamificación: expectativa educativa, impacto en la salud}

\section{Resumen:}

Los dispositivos tecnológicos forman parte de la vida cotidiana de la sociedad y también están presentes en el entorno escolar. Por lo tanto, la gamificación se ha utilizado como una alternativa a los enfoques tradicionales de la educación. Así, se llevó a cabo un ensayo teórico con el objetivo de reflexionar sobre la gamificación en la educación y el riesgo del uso excesivo de juegos digitales para la salud de los adolescentes. Esta reflexión analiza el uso de juegos digitales en el contexto educativo, el riesgo detrás de los juegos digitales y las estrategias para minimizar el daño a la salud, derivado del uso de juegos. Se llegó a la conclusión de que la gamificación resulta ser un excelente catalizador para el aprendizaje. Sin embargo, si se estimula sin moderación, puede generar una fascinación por el juego, desencadenando sentimientos de ansiedad, agresión y compulsión. Por lo tanto, es necesario que los agentes sociales directa o indirectamente involucrados en la educación de los adolescentes, como la familia, la comunidad escolar y los profesionales de la salud, sean conscientes de los posibles cambios en el comportamiento de los adolescentes y estructurar acciones, estrategias y políticas de atención de la salud, enfocados en el uso de juegos digitales por parte de los adolescentes.

Palabras clave: Educación, Juegos de video, Materiales de enseñanza, Salud del adolescente.

\section{INTRODUÇÃo}

A expansão tecnológica e o desenvolvimento dos meios de comunicação e das conexões sem fio possibilitaram às pessoas utilizarem diferentes acessórios para a execução das mais variadas tarefas, tais como localizar-se geograficamente, buscar meios de diversão e realizar compras. Estes dispositivos tecnológicos que fazem parte do cotidiano da sociedade marcam presença também no ambiente escolar, sendo possível envolvê-los na educação (CANI et al., 2017).

No caso dos adolescentes, a Pesquisa Nacional por Amostras de Domicílio (PNAD) realizada em 2015, demonstrou que jovens na faixa etária entre 15 e 17 anos, 18 e 19 anos constituem-se como o grupo de pessoas que mais acessam a internet, com percentuais equivalentes a 82,0\% e 82,9\%, respectivamente (IBGE, 2016). Logo o espaço virtual é parte da rotina diária deste grupo de pessoas. 
O principal local de acesso à internet destes adolescentes é o próprio domicílio, mas isso também ocorre na casa de amigos, em lan houses, na escola e pelo celular. O tempo gasto navegando na internet é de duas a três horas diárias, em média. As ferramentas mais utilizadas são as redes sociais, correio eletrônico, jogos on-line e aplicativos de mensagens instantâneas (BRAGA; SILVA, 2017).

Frente a este novo contexto, a postura pedagógica de muitos professores vem mudando, pois percebeu-se que aulas tradicionais, fragmentadas e descontextualizadas visam apenas a memorização. Portanto, relegam a um segundo plano atitudes articuladas e significativas, mostrando-se desinteressantes aos olhos dos alunos, pois eles não veem sentido no que estão aprendendo (REIS; BITENCOURT, 2016).

Diante disso, buscando tornar suas aulas mais dinâmicas, muitos professores investiram em novas metodologias, de forma a incluir artefatos que fazem parte do cotidiano dos estudantes no contexto da sala de aula. Esta nova perspectiva de ensino vem sendo evidenciada em pesquisas sobre a prática docente, mediante a divulgação de metodologias que adotam ferramentas tecnológicas mais atrativas aos alunos, dentre as quais destaca-se $o$ uso de jogos digitais (COSTA; SOUZA, 2015; SILVA; FARIAS JUNIOR; LEMOINE NEVES, 2016).

Estudos demonstraram que os jogos digitais vêm sendo utilizados e têm apresentado bons resultados, em distintas áreas de conhecimento, como história, matemática, química e línguas estrangeiras (VALLE, 2014; COSTA; SOUZA, 2015; SILVA; FARIAS JUNIOR; LEMOINE NEVES, 2016; CANI et al., 2017). Estes resultados são decorrentes de estratégias empregadas nos jogos, que estimulam o jogador a realizar tarefas em um ambiente lúdico e divertido e, portanto, funcionam como um catalizador para a aprendizagem. Este fenômeno recebe o nome de gamificação (CANI et al., 2017).

A gamificação tornou-se um fenômeno cultural e tem sido utilizada como alternativa às abordagens tradicionais da educação. Seu potencial para promover a aprendizagem foi reconhecido pelo Ministério da Educação, que credenciou uma plataforma on-line para que os candidatos ao Exame Nacional do Ensino Médio (ENEM) pudessem se preparar para as provas, mediante a resolução de desafios (CANI et al., 2017).

Assim, neste ensaio teórico objetivou-se refletir sobre a gamificação na educação e sobre o risco que o uso desmedido dos jogos digitais representa para a saúde dos adolescentes. 


\section{OS JOGOS DIGITAIS NO CONTEXTO EDUCACIONAL}

Mesmo com todas as inovações tecnológicas, temos que pensar que alguns professores se baseiam somente na transmissão da informação. Desconsideram as experiências vividas pelos discentes, limitando sua criatividade, o que torna o processo de aprendizagem falho (RAMALHO; SIMÃO; PAULO, 2014). Além do mais, a escola parece ter criado um abismo entre aprendizagem e saber, não podendo ocorrer aprendizagem e diversão juntas.

No entanto, os jogos digitais vêm justamente para mostrar o contrário, pois a sensação de prazer a que os jogadores são submetidos pode provocar um aprendizado involuntário, sem compromisso, sem pressão, despertando o interesse dos alunos por meio de uma metodologia envolvente, lúdica e desafiadora, que amplia as características humanas motora, afetiva, moral e cognitiva (RAMALHO; SIMÃO; PAULO, 2014; HOFFMANN; BARBOSA; SANTOS, 2016).

Os jogos são destacados nos Parâmetros Curriculares Nacionais (PCNs) como artefatos promotores do desenvolvimento, sendo citados em diferentes áreas e disciplinas, mediante articulação entre o conhecimento e o imaginário (REIS; BITENCOURT, 2016). Assim, os jogos possibilitam a aprendizagem ativa, por meio do estímulo ao pensamento crítico, ao desenvolvimento de capacidades de interação, à negociação de informações e resolução de problemas; sendo que a aprendizagem cooperativa implica em maior responsabilidade na aprendizagem, levando os estudantes à assimilação de conceitos e à construção mais autônoma do conhecimento (ALMEIDA et al., 2016).

Ressalta-se também que, por meio dos jogos, é possível abordar o conteúdo de forma diferenciada, proporcionando a tomada de decisões, o raciocínio lógico, a análise dos resultados, além de revisitar conceitos e objetivos, reformular os procedimentos praticados durante o jogo, bem como aprender com os erros cometidos, sem que ocorram frustrações, que estimula os estudantes a realizarem novas tentativas (HOFFMANN; BARBOSA; SANTOS, 2016). Do ponto de vista de professores, os jogos facilitam a apropriação do conteúdo mediante o desenvolvimento de estratégias que incidem sobre o processo de aprendizagem, tais como a resolução de problemas, raciocínio dedutivo e memorização (REIS; BITENCOURT, 2016).

Os docentes também reconhecem a necessidade da própria alfabetização digital, para 
poder exercer seu papel de mediador, ou seja, aquele que orienta e incentiva os discentes no uso desta tecnologia (RAMALHO; SIMÃO; PAULO, 2014). Além disso, devem estar atentos ao planejamento dos métodos, pois esta prática requer tanto planejamento pedagógico quanto tecnológico, visto que, para o empreendimento ser bem-sucedido, os jogadores devem dominar as regras e estar conscientes do objetivo do jogo e do que suas decisões podem acarretar (HOFFMANN; BARBOSA; SANTOS, 2016).

Coopera para isso a facilidade que os jovens têm de adaptar-se aos dispositivos móveis, utilizando seus recursos para realizar diferentes tarefas, sendo uma delas o próprio jogo (ALMEIDA et al., 2016). Diante desta perspectiva o professor pode lançar mão da gamificação para ensinar e o aluno aprender, utilizando o próprio celular, seja na sala de aula, no pátio da escola, em casa, ou na casa de amigos.

No processo de gamificação podem ser usados jogos de diferentes estilos como o roleplaying game (RPG) e os jogos de simulação. No RPG, o jogador incorpora um personagem do enredo, toma decisões, assume responsabilidades e age de acordo com as regras do jogo. Já a evolução do personagem é vista em cada evento, pois no jogo não há níveis nem fases. 0 jogo conta com um relógio em tempo real, que contribui para que algumas ações sejam completadas apenas durante um horário específico, como se fosse a vida real, fato que torna o jogo longo e complexo, exigindo dedicação para ser completado. No que tange à educação este jogo permite ao aluno se divertir e aprender ao mesmo tempo (RAMALHO; SIMÃO; PAULO, 2014).

Nos jogos de simulação visa-se medir e desenvolver habilidades de gerenciamento e construção do jogador, podendo ser necessário desenvolver estratégias conforme a sua jogabilidade. Os simuladores apresentam problemas para o jogador resolver, mas também desenvolvem habilidades para que seja possível resolvê-los, sendo essa metodologia baseada nas capacidades cognitivas. Sua importância para a educação está no fato de desenvolver habilidades, testar e corrigir, sem marcar o aluno negativamente, por suas escolhas erradas (RAMALHO; SIMÃO; PAULO, 2014).

Um exemplo de simulado é o Quiz, que se constitui como um jogo de questões sobre determinado assunto, onde o estimulo é desencadeado pela pergunta, e o incentivo pela resposta que, sendo correta, confere aumento na pontuação do jogador, além de considerar o tempo de resposta, pois quanto menor, maior é a pontuação atribuída ao jogador. Este jogo 
pode ser utilizado como forma de memorização e de avaliação. Nesse viés avaliativo, o Quiz apresentou bons resultados, pois a turma que foi submetida a esta nova forma de avaliação mostrou-se feliz e empolgada, situação não observada nas avaliações tradicionais (ALMEIDA et al., 2016). O Quiz é o mesmo jogo disposto na plataforma preparatória para o ENEM, avalizado pelo Ministério da Educação.

Sobre os benefícios de utilizar os jogos na educação podemos destacar a aprendizagem lúdica, a capacidade de simulação e de organizar elementos para atingir um objetivo, o enfrentamento de situações problema, a definição de estratégias cooperativas e as próprias descobertas (RAMALHO; SIMÃO; PAULO, 2014). Ressalta-se que os jogos disputados em plataformas on-line favorecem a tomada de decisão e o raciocínio crítico. Nesses jogos, a aprendizagem por descobertas se dá pelo feedback imediato; o aprendizado de competências, pela necessidade de conhecimentos associados com as identidades dos personagens; a socialização, pela interação e colaboração com outros jogadores. Há, ainda, o desenvolvimento da coordenação motora, de habilidades espaciais e comportamento expert, uma vez que alguns estudantes se tornam-se profundos conhecedores dos temas abordados no jogo (REIS; BITENCOURT, 2016).

Não há dúvidas sobre as possibilidades de aprendizagem que a gamificação favorece, mas é preciso pensar que existe uma grande polêmica em torno dos games, devido ao conteúdo violento presente em muitos deles. A mídia tem apresentado atos de violência cometido por jovens, os quais teriam sido inspirados no conteúdo dos jogos (REIS; BITENCOURT, 2016). Vale destacar que tal comportamento está relacionado a questões afetivas e socioeconômicas, devendo a violência ser analisada a partir dos fatores que cercam a vida dos jogadores.

\section{O RISCO POR TRÁS DOS JOGOS DIGITAIS}

Não há dúvidas quanto às vantagens da gamificação, mas percebe-se alguns riscos envolvendo o estímulo do uso dos jogos digitais. Estes riscos vão desde a naturalização da violência até o desenvolvimento de comportamentos aditivos. Esta percepção é comum durante a prática docente, pois sempre que possível a grande maioria dos adolescentes 
prioriza os jogos à interação social, por meio da comunicação verbal.

As crianças e os adolescentes de hoje são nativos digitais, pois nasceram numa época tecnológica e, portanto, sempre estiveram em contato com ela. Isso faz com que seu envolvimento e manuseio lhes seja natural (REIS; BITENCOURT, 2016). Como já mencionado, o uso da internet é constante na vida dos adolescentes, sendo que um dos motivos que os levam a conectar- se é a utilização de jogos digitais $(28,2 \%)$, sendo estes jogos de diferentes tipos ou formatos, utilizados individualmente ou em grupos (RIAL et al., 2014).

Os jogos comportam um sistema em que os jogadores entram voluntariamente e se engajam em um conflito artificial que é definido por regras e têm seus resultados quantificáveis. Todos eles estão subsidiados nos seguintes elementos: a) Representação: o jogo edifica-se sobre uma representação gráfica da realidade, apresenta um conjunto de normas e objetivos específicos; b) Interação: está focada na forma como a representação da realidade se altera e possibilita a ação do jogador, bem como ele interage com outros jogadores; c) Conflito: surge naturalmente quando há interação do jogador; e d) Segurança: o conflito geralmente cria um cenário que oferece riscos/perigo ao jogador, mas apesar do jogador sujeitar-se à experiência psicológica do conflito e do perigo, não ocorre danos físicos, possibilitando a dissociação das consequências das ações (BREDA et al., 2014; REIS; BITENCOURT, 2016).

Deste modo, o jogo edifica-se como meio de obter prazer e inclusão social, pois, ao jogar, o adolescente é conduzido a uma realidade paralela à sua, a qual mantem o caráter de uma fantasia lúdica, que lhe permite a significação e ressignificação de conceitos (CAVALLI; TREVISOL; VENDRAME, 2013).

Convém ressaltar que a utilização dos jogos on-line, em sua maioria, deve-se à possibilidade de domínio, autodeterminação, estética agradável e fantástica, e feedback da ação, o que pode levar à necessidade imperiosa de jogar (MARCO; CHÓLIZ, 2017). Deste modo, o incentivo à gamificação na escola, mesmo com fins de aprendizagem, pode estimular os jovens a manterem-se ativos no jogo, tornando-se prejudicial.

Corroboram para isso as plataformas on-line, porque proporcionam alto grau de interatividade entre os jogadores, mesmo que estes não estejam no mesmo espaço físico. A interatividade, por sua vez, passa a ser uma constante, pois alguns jogos permanecem em andamento ininterruptamente, mesmo seu praticante estando ausente. Diante disso, é 
necessário que o jogador se mantenha online o maior tempo possível, para evitar ser superado por seus adversários (BREDA et al., 2014).

A permanência da conexão desmedida ao ambiente virtual, para jogar, pode gerar a dependência/vício. Esta situação está descrita no Manual Diagnóstico e Estatístico dos Transtornos Mentais/DMS-5 como "Internet Gaming Disorder", sendo necessário prejuízo ou sofrimento significativos, indicados pela presença de cinco ou mais dos sintomas abaixo relacionados, durante um período de 12 meses: preocupação com jogos on-line (o indivíduo pensa constantemente sobre o jogo, que se torna a atividade mais relevante na sua vida); sintomas de abstinência quando a pessoa se afasta do jogo (esses sintomas são tipicamente descritos como irritabilidade, ansiedade ou tristeza, mas sem os sinais físicos de abstinência); tolerância - necessidade de passar cada vez mais tempo jogando on-line; tentativas fracassadas de controlar sua participação nos jogos; perda de interesse em hobbies e entretenimentos anteriores, como consequência dos jogos online; manutenção do uso excessivo de jogos on-line, apesar do reconhecimento de problemas psicossociais; mentir para familiares, terapeutas e outros, com relação ao tempo utilizado nos jogos; uso dos jogos on-line para escapar ou aliviar um humor "negativo" (por exemplo, sentimento de impotência, culpa, ansiedade); colocar em risco ou ter perda significativa de um relacionamento, trabalho, ensino ou oportunidade de carreira, por causa do envolvimento com jogos on-line (BREDA et al., 2014; MARCO; CHÓLIZ, 2017).

A dependência do jogo pode acarretar isolamento social, alteração do padrão do sono, deficiência nutricional, deficiência de higiene, alterações de humor, sintomas de abstinência entre outros. Nos casos mais graves, pode chegar a comportamentos suicidas e homicidas. Tais problemas tendem a manter-se nos jogadores compulsivos, revelando a existência de comorbidades psiquiátricas associadas ao jogo (MARCO; CHÓLIZ, 2017).

Entre as comorbidades evidenciadas nos indivíduos com esse tipo de dependência estão a depressão, ansiedade social e transtorno de déficit de atenção/hiperatividade. Geralmente são jovens mais vulneráveis que, devido ao seu alto grau de impulsividade, possuem menor habilidade social, empatia e controle emocional. Além disso, o adolescente que apresenta dependência por jogos passa muito tempo jogando, afastando-se da família e dos amigos, o que agrava o nível de isolamento social (BREDA et al., 2014). Outra comorbidade associada ao vício em jogos eletrônicos é a obesidade, pois estes indivíduos têm seu gasto 
calórico reduzido e consomem excessivamente alimentos de baixa qualidade nutricional e ricos em calorias, o que resulta em sobrepeso, que favorece o agravamento dos estados de depressão e ansiedade social (BREDA et al., 2014).

Os jogos utilizados pelos adolescentes são: esportes, tiro, ação, luta e estratégia. Destes, são considerados como violentos pelos adolescentes os jogos que envolvem tiro $(64,43 \%)$, ação, luta e estratégia $(28,03 \%)$ e esporte $(1,89 \%)$. Salienta-se também que os próprios adolescentes relatam utilizar os jogos como forma de diversão e de extravasar sentimentos (CAVALLI; TREVISOL; VENDRAME, 2013). Contudo não parecem perceber que, no uso de jogos violentos, pode estar incutida a necessidade de satisfazer vontades latentes, que podem se transformar em comportamentos violentos.

Contrapondo o pensamento de Reis e Bitencourt (2016), citados anteriormente, o efeito negativo do conteúdo violento de alguns jogos foi confirmado por estudos experimentais e observacionais. Nestes estudos observou-se o desenvolvimento de comportamentos, pensamentos e sentimentos agressivos em seus jogadores. Também foi observada excitação psicofisiológica, redução da empatia e do comportamento pró-social, em curto e longos prazos (BREDA et al., 2014).

Estudos de neuroimagem demonstraram que, ao se confrontar frequentemente com cenas de violência (comuns em jogos de tiro), pode-se criar uma habituação aos efeitos dos estímulos aversivos, visto que ocorre redução na ativação frontal do cérebro e consequente criação de um mecanismo de dessensibilização, que potencializa a prática contínua de jogos eletrônicos, de forma patológica (LEMOS et al., 2014). Deste modo, o uso excessivo e repetitivo de jogos com conteúdo violento pode funcionar como estimulante de agressividade em crianças e adolescentes.

Acerca da questão familiar é preciso mencionar que nem sempre os pais estão conscientes dos riscos aos quais seus filhos estão expostos, ao navegar na internet, pois conforme demonstrado por Livingstone et al (2011), apenas $28 \%$ dos pais bloqueiam ou filtram sites e apenas $23 \%$ observam os sites visitados pelos filhos. Portanto, entende-se que os pais desconhecem o conteúdo presente nos sites que seus filhos acessam, bem como os efeitos de alguns jogos sobre a saúde dos mesmos.

A postura dos pais pode ser explicada pela cultura de permissividade vigente, a qual é 
fruto das distorções referentes às atitudes dos pais e da interpretação equivocada referente à seguridade em aspectos biopsicossociais, conforme o Estatuto da Criança e do Adolescente (ECA). Outra explicação para o comportamento dos pais é a ilusão de que seus filhos estão seguros em casa, diante do computador, o que denota desconhecimento do risco que está nas associações e leituras que os adolescentes fazem do conteúdo exposto nos jogos (CAVALLI; TREVISOL; VENDRAME, 2013).

Diante deste quadro, é preciso pensar em estratégias que venham a possibilitar ações que minimizem as consequências do uso excessivo dos jogos digitais.

\section{ESTRATÉGIAS PARA MINIMIZAR DANOS À SAÚDE DECORRENTES DO USO DE JOGOS DIGITAIS}

Na perspectiva de promover a saúde de crianças e adolescentes brasileiros foi criado, em 2007, o Programa de Saúde na Escola (PSE), uma política intersetorial que pontua a integração e articulação permanente da educação e da saúde. Sendo assim, o PSE visa contribuir para o fortalecimento de ações que proporcionem a participação da comunidade escolar em programas e projetos que articulem saúde e educação, para o enfrentamento das vulnerabilidades que comprometem o pleno desenvolvimento de crianças, adolescentes e jovens brasileiros (BRASIL, 2011).

Podem participar do PSE todos os municípios brasileiros, todas as equipes de Atenção Básica, Escolas de Ensino no Fundamental e Médio, creches e pré-escolas. Portanto, o programa contempla todos os níveis de ensino. Para participar do PSE é necessário que a escola/pré-escola/creche seja incluída, mediante cadastro on-line. No Rio Grande do Sul, por exemplo, o contingente total de escolas de educação básica cadastradas é de 5.562, com 1.249.242 alunos pactuados e 448.662 equipes pactuadas, valores que são referentes a 486 municípios. Em se tratando do contexto local, atualmente existem 109 escolas cadastradas, com 36.727 alunos pactuados e 75 equipes pactuadas (BRASIL, 2021).

Destaca-se que, no momento da adesão, as ações do PSE devem ser pactuadas entre as instituições de ensino e de saúde, não sendo possível exclui-las ou alterá-las. Contudo, mediante o diagnóstico local, a gestão do município pode definir e acrescentar novas ações 
para serem realizadas, devendo informá-las no sistema e-Gestor, no momento da adesão. Atualmente estão vigentes as seguintes ações: Combate ao mosquito Aedes aegypti; Promoção da segurança alimentar e nutricional e da alimentação saudável e combate à obesidade infantil; Direito sexual e reprodutivo e prevenção de DST/AIDS; Prevenção ao uso de álcool, tabaco, crack e outras drogas; Promoção da Cultura de Paz, Cidadania e Direitos Humanos; Promoção das práticas Corporais, da Atividade Física e do lazer nas escolas; Prevenção das violências e dos acidentes; Identificação de estudantes com possíveis sinais de agravos de doenças em eliminação; Promoção e Avaliação de Saúde bucal e aplicação tópica de flúor; Verificação da situação vacinal; Promoção da saúde auditiva e identificação de estudantes com possíveis sinais de alteração; e Promoção da saúde ocular e identificação de com possíveis sinais de alteração (BRASIL, 2018).

Assim, sob a perspectiva do risco de consequências negativas para a saúde dos adolescentes, decorrentes do estímulo à gamificação, é importante que sejam desenvolvidas parcerias da rede de ensino com a rede básica de saúde, para promover a saúde na escola. Dentre os sinais que podem indicar prejuízos à saúde dos adolescentes destacam-se o conteúdo violento de alguns jogos, o tempo de uso e o tipo de jogo que os alunos estão utilizando, bem como prejuízos relacionados à qualidade do sono, queda no rendimento escolar, no padrão nutricional e na higiene corporal, decréscimo na interação social e padrões de isolamento, autolesão, sentimentos de ansiedade e agressividade, entre outros, cuja prevenção apresentam ligação com algumas das ações do PSE.

Nesta condição, é urgente implementar oficinas para identificar o simbolismo do conteúdo dos jogos para os adolescentes; discutir estes significados com e para a comunidade escolar; conhecer os motivos que levam os adolescentes a jogar; agir sobre estes significados, visando sua correção ou minimização; minimizar ações de violência entre os estudantes e na escola; envolver os pais e responsáveis, buscando apoio e orientando-os para os riscos de adição pelo uso excessivo dos jogos virtuais; reduzir o tempo de jogo; envolver os alunos em outras atividades interativas, como esportes praticados em grupo; e encaminhar os estudantes com comprometimento de saúde para redes de apoio. 


\section{CONSIDERAÇÕES FINAIS}

É fato que o mundo tecnológico faz parte do cotidiano dos adolescentes e os métodos pedagógicos que utilizam tais ferramentas lhes são mais atrativos. A gamificação demonstra ser um excelente catalizador da aprendizagem, pois possibilita a ressignificação de conceitos, devido à interação que propicia entre o objeto e o aprendiz.

Ao jogar, o adolescente é reportado a uma realidade paralela à sua, na qual vivencia situações psicológicas que lhe propiciam prazer. No entanto, isso pode gerar um fascínio pelo jogo, desencadeando sentimentos de ansiedade, agressividade e de compulsão. Estes sentimentos afetam sua saúde de forma negativa. Logo, o uso de jogos digitais deve ser monitorado, tanto pela família, quanto pelos educadores.

Além disso, a comunidade escolar deve ficar atenta para possíveis mudanças no comportamento dos adolescentes e buscar parcerias com a rede básica de saúde, de modo a desenvolver estratégias focadas nesta temática, no intuito de manter e promover a sua saúde dos escolares.

\section{REFERÊNCIAS}

ALMEIDA, Danielly Silva Ramos; FERREIRA, Michelly Carvalho; SOUSA, Claudia Niedes da Silva; DIAS, Márcia Adelino da Silva. O USO DE JOGOS DIGITAIS COMO INSTRUMENTO AVALIATIVO DA APRENDIZAGEM DO ENSINO DE BIOLOGIA: um relato de experiência. $2016 . \quad$ Disponível em: <https://www.editorarealize.com.br/index.php/artigo/visualizar/23164>. Acesso em: 10 jul. 2021.

BRAGA, Wylnara dos Santos; SILVA, Bento Duarte da. Social x Digital: uma análise do uso das tic no contexto escolar. Revista de Estudios e Investigación En Psicología y Educación, [S.L.], p. 119-123, 17 dez. 2017. Universidade da Coruna. Disponível em: <http://dx.doi.org/10.17979/reipe.2017.0.13.2536>. Acesso em: 10 jul. 2021.

BRASIL. Documento Orientador: Indicadores e Padrões de Avaliação - PSE Ciclo 2017/2018. 2017. Disponível em: <http://189.28.128.100/dab/docs/portaldab/documentos/documento_orientador_monitoramento_pse_2017_2018.p df>. Acesso em: 10 jul. 2021.

BRASIL. Passo a Passo PSE Programa de Saúde na Escola. 2011. Disponível em: <https://bvsms.saude.gov.br/bvs/publicacoes/passo_a_passo_programa_saude_escola.pdf>. Acesso em: 10 jul. 2021.

BRASIL. Painel de Adesões Programa Saúde na Escola - Ciclo 2021-2022. 2021. Disponível em: <https://sisaps.saude.gov.br/pse/relatorio>. Acesso em: 10 jul. 2021.

BREDA, Vitor Carlos Thumé; PICON, Felipe Almeida; MOREIRA, Laura Magalhães; SPRITZER, Daniel Tornaim. Dependência de Jogos Eletrônicos em Crianças e Adolescentes. Revista Brasileira de Psicoterapia, Porto Alegre, v. 16, n. 1, p. 53-67, jan. 2014. Disponível em: <https://cdn.publisher.gn1.link/rbp.celg.org.br/pdf/v16n1a06.pdf>. Acesso em: 10 jul. 2021.

CANI, Josiane Brunetti; PINHEIRO, Ivana Queiroz; SANTIAGO, Maria Elizabete Villela; SOARES, Gilvan Mateus. Análise de jogos digitais em dispositivos móveis para aprendizagem de línguas estrangeiras. Revista Brasileira de 
Linguística Aplicada, [S.L.], v. 17, n. 3, p. 455-481, set. 2017. FapUNIFESP (SciELO). Disponível em: <http://dx.doi.org/10.1590/1984-6398201711880>. Acesso em: 10 jul. 2021.

CAVALLI, Franciely da Silva; TREVISO, Maria Teresa Ceron; VENDRAME, Thiago. Influência dos jogos eletrônicos e virtuais no comportamento social dos adolescentes. Psicol. Argum, Curitiba, v. 72, n. 31, p. 155-163, jan. 2013. Disponível em: <https://periodicos.pucpr.br/index.php/psicologiaargumento/article/view/20467/19725>. Acesso em: 10 jul. 2021.

COSTA, Mylani Nathalini Dantas; SOUZA, Daniel Faustino Lacerda de. JOGOS DIGITAIS: uma alternativa metodológica para o ensino de matemática. Revista Internacional Interdiscilpinar: REDIN, Taquara, v. 4, n. 1, p. 1-10, nov. 2015. Disponível em: <https://seer.faccat.br/index.php/redin/article/view/279>. Acesso em: 10 jul. 2021.

HOFFMANN, Me. Luís Fernando; BARBOSA, Débora Nice Ferrari; SANTOS, Paulo Ricardo dos. Aprendizagem baseada em jogos digitais educativos para o ensino da matemática - um estudo-piloto a partir da utilização do Erudito. Teknos Revista Científica, [S.L.], v. 16, n. 2, p. 38, 30 dez. 2016. Fundacion Universitaria Tecnologico de Comfenalco - FUTC. Disponível em: <http://dx.doi.org/10.25044/25392190.820>. Acesso em: 10 jul. 2021.

IBGE. Pesquisa nacional por amostra de domicílios: síntese de indicadores 2015. 2016. Disponível em: <https://biblioteca.ibge.gov.br/visualizacao/livros/liv98887.pdf>. Acesso em: 10 jul. 2021.

LEMOS, Igor Lins; DINIZ, Paula Rejane Beserra; PERES, Julio Fernando Prieto; SOUGEY, Everton Botelho. Neuroimagem na dependência de jogos eletrônicos: uma revisão sistemática. Jornal Brasileiro de Psiquiatria, [S.L.], v. 63, n. 1, p. 57-71, mar. 2014. FapUNIFESP (SciELO). Disponível em: <http://dx.doi.org/10.1590/0047-2085000000008>. Acesso em: 10 jul. 2021.

LIVINGSTONE, Sonia; HADDON, Leslie; GÖRZIG, Anke; ÓLAFSSON, Kjartan. Risks and safety on the internet: the perspective of European children: full findings and policy implications from the EU Kids Online survey of 916 year olds and their parents in 25 countries. 2011. Disponível em: <http://eprints.lse.ac.uk/33731/1/Risks\%20and\%20safety\%20on\%20the\%20internet(lsero).pdf>. Acesso em: 10 jul. 2021.

MARCO, Clara; CHÓLIZ, Mariano. Eficacia de las técnicas de control de la impulsividad en la prevención de la adicción a videojuegos. Terapia Psicológica, Santiago, v. 35, n. 1, p. 57-69, 2017. Disponível em: <https://www.scielo.cl/pdf/terpsicol/v35n1/art06.pdf>. Acesso em: 10 jul. 2021.

RAMALHO, John Eric; SIMÃO, Fábio; PAULO, Andrea Barbosa Delfini. APRENDIZAGEM POR MEIO DE JOGOS DIGITAIS: um estudo de caso do jogo animal crossing. Revista Ensaios Pedagógicos, Curitiba, v. 7, n. 2, p. 1-13, dez. 2014. Disponível em: <https://www.opet.com.br/faculdade/revista-pedagogia/pdf/n8/artigo-4.pdf>. Acesso em: 10 jul. 2021.

REIS, Fernanda de Melo; BITENCOURT, Ricardo Barbosa. Games no Ensino de História: aplicação na disciplina de história no ensino fundamental. Aplicação na Disciplina de História no Ensino Fundamental. 2016. Disponível em: <http://www.sbgames.org/sbgames2016/downloads/anais/157378.pdf>. Acesso em: 10 jul. 2021.

RIAL, Antonio; GÓMEZ, Patricia; BRAÑA, Teresa; VARELA, Jesús. Actitudes, percepciones y uso de Internet y las redes sociales entre los adolescentes de la comunidad gallega (España). Anales de Psicología, Murcia, v. 30, n. 2, p. 642-655, maio 2014. Disponível em: <https://scielo.isciii.es/pdf/ap/v30n2/psico_adolescencia5.pdf>. Acesso em: 10 jul. 2021.

SILVA, Ysabele C. O. da; FARIAS JUNIOR, Ivaldir de; NEVES, Juliana K. A. Lemoine. JOGOS DIGITAIS COMO ESTRATÉGIA PEDAGÓGICA. Revista Eletrônica Estácio Recife, Recife, v. 1, n. 1, p. 1-11, jul. 2016. Disponível em: <https://reer.emnuvens.com.br/reer/article/view/83>. Acesso em: 10 jul. 2021.

VALLE, Daniel Simões do. Os jogos nas aulas de História: reflexões acerca dos saberes e das práticas docentes. reflexões acerca dos saberes e das práticas docentes. 2014. Disponível em: <http://www.encontro2014.rj.anpuh.org/resources/anais/28/1400280507_ARQUIVO_ArtigoparaAnpuhregional2014 -OsjogosnasaulasdeHistoria.pdf>. Acesso em: 10 jul. 2021.

\section{(c) $\mathbf{E Y}$}

Este trabalho está licenciado com uma Licença Creative Commons - Atribuição 4.0 Internacional. 